\title{
Establishing Robust Stability of Discrete-Time Systems with Time-Varying Uncertainty: the Gram-SOS Approach
}

\author{
Graziano Chesi \\ Department of Electrical and Electronic Engineering \\ The University of Hong Kong \\ Contact: http://www.eee.hku.hk/ ${ }^{\sim}$ chesi
}

\begin{abstract}
This paper addresses the problem of establishing robust asymptotical stability of discrete-time linear systems polynomially affected by time-varying uncertainty confined into a polytope. A linear matrix inequality (LMI) condition for establishing robust asymptotical stability is proposed by introducing a novel approach for establishing the existence of a common homogeneous polynomial Lyapunov function (HPLF). This approach consists, firstly, of introducing a Gram matrix built with respect to the state and parametrized by an arbitrary vector function of the uncertainty, and secondly, of requiring that a transformation of the introduced Gram matrix is a sum of squares (SOS) of matrix polynomials. The approach, hence, is referred to as a Gram-SOS approach. It is shown that the proposed LMI condition is sufficient for any degree of the HPLF candidate, that includes quadratic robust stability as a special case, and that is also necessary for a sufficiently large degree of the HPLF candidate. Numerical examples also show that the proposed LMI condition can outperform alternative ones in terms of conservatism and computational burden.
\end{abstract}

\section{Introduction}

A fundamental problem in systems with uncertainty amounts to establishing whether a linear system affected by uncertain parameters is asymptotically stable for all the admissible values of the parameters. Numerous works have been proposed in the literature for addressing this problem, which can be classified according to different criteria, e.g. based on the nature of 
the system (such as continuous-time or discrete-time), type of uncertainty (such as time-invariant or time-varying), dependence of the coefficients of the system on the uncertainty (such as linear or rational), and shape of the set of admissible uncertainty (such as multi-interval or polytopic). See e.g. [1-3].

For continuous-time linear systems, various methods have been developed, typically focusing on systems where the coefficients depend linearly on the uncertainty and the uncertainty is confined into a polytope. These methods are generally based on the search for a suitable Lyapunov function, and the type of uncertainty characterizes the type of Lyapunov function that is searched for. Specifically, in the case of time-invariant uncertainty, pioneering methods have searched for a quadratic Lyapunov function, see e.g. [4], and more recent ones have proposed the use of parameter-dependent quadratic Lyapunov functions in order to reduce the conservatism, see e.g. [2]. Then, in the case of time-varying uncertainty, results obtained with common quadratic Lyapunov functions have been improved by considering common nonquadratic Lyapunov functions. See e.g. [5-8] where piecewise quadratic Lyapunov functions are searched for, [9-11] where the use of polyhedral and smoothed polyhedral Lyapunov functions is investigated, and $[2,12-15]$ which address the construction of homogeneous polynomial Lyapunov functions (HPLFs).

For discrete-time linear systems, analogous methods have been developed in the case of time-invariant uncertainty, see e.g. [2,16-18]. However, the case of time-varying uncertainty has been less investigated. Indeed, contrary to continuous-time systems with linear dependence on the uncertainty where the time derivative of a Lyapunov function candidate is linear in the uncertainty, one has that the time difference of such a candidate is nonlinear in the uncertainty for discrete-time systems and checking the vertices does not suffice to ensure robust stability. Existing works include [19-21] where robust stability and robust stabilization are investigated through quadratic Lyapunov functions, set-induced Lyapunov functions, and parameter-dependent quadratic Lyapunov functions, respectively.

This paper addresses the problem of establishing robust asymptotical stability of discrete-time linear systems affected by time-varying structured uncertainty. Specifically, it is supposed that the coefficients of the system depend polynomially on the uncertainty, and that the uncertainty is confined into a polytope. A linear matrix inequality (LMI) condition for establishing robust asymptotical stability is proposed by introducing a novel approach for establishing the existence of a common HPLF. This approach consists, firstly, of introducing a Gram matrix built with respect to the state and 
parametrized by an arbitrary vector function of the uncertainty, and secondly, of requiring that a transformation of the introduced Gram matrix is a sum of squares (SOS) of matrix polynomials. The approach, hence, is referred to as a Gram-SOS approach. It is shown that the proposed LMI condition is sufficient for any degree of the HPLF candidate, that includes quadratic robust stability as a special case, and that is also necessary for a sufficiently large degree of the HPLF candidate. A preliminary version of this paper, without Theorem 3 (which investigates the necessity of the condition) and without Theorem 4 (which investigates the reduction of the computational burden) appeared as reported in [22].

The paper is organized as follows. Section 2 introduces the preliminaries. Section 3 describes the proposed approach. Section 4 investigates its conservatism. Section 5 addresses the reduction of its computational burden. Section 6 presents some illustrative examples. Lastly, Section 7 concludes the paper with some final remarks.

\section{Preliminaries}

\subsection{Problem Formulation}

Notation: $\mathbb{R}$ : real number set; $0_{n}$ : origin of $\mathbb{R}^{n} ; \mathbb{R}_{0}^{n}: \mathbb{R}^{n} \backslash\left\{0_{n}\right\} ; I$ : identity matrix; $a^{2}$ with $a=\left(a_{1}, a_{2}, \ldots\right)^{\prime}:\left(a_{1}^{2}, a_{2}^{2}, \ldots\right)^{\prime} ; A^{\prime}$ : transpose of $A ; A>0$, $A \geq 0$ : symmetric positive definite and symmetric positive semidefinite matrix $A ; A^{\otimes m}: m$-th Kronecker power of $A ; \operatorname{conv}\{A, B, \ldots\}$ : convex hull of $A, B, \ldots ; \lambda_{\min }(A)$ : minimum eigenvalue of $A ; *$ : corresponding block in symmetric matrices.

We consider discrete-time linear systems polynomially affected by timevarying uncertainty confined into a polytope in the canonical form

$$
\left\{\begin{array}{l}
x(t+1)=A(s(t)) x(t) \\
s(t) \in \mathcal{S}
\end{array}\right.
$$

where $x(t) \in \mathbb{R}^{n}$ is the state, $s(t) \in \mathbb{R}^{r}$ is the time-varying uncertainty, $A: \mathbb{R}^{r} \rightarrow \mathbb{R}^{n \times n}$ is a matrix homogeneous polynomial of degree $d$, i.e.,

$$
A(s)=\sum_{\substack{i_{1}+\ldots, i_{r}=d \\ i_{1} \geq 0, \ldots, i_{r} \geq 0}} A_{i_{1}, \ldots, i_{r}} \prod_{j=1}^{r} s_{j}^{i_{j}}
$$

for some given matrices $A_{i_{1}, \ldots, i_{r}} \in \mathbb{R}^{n \times n}$, and $\mathcal{S}$ is the simplex of dimension $r$, i.e.

$$
\mathcal{S}=\left\{s \in \mathbb{R}^{r}: o(s)=1, s_{i} \geq 0\right\}
$$


where $o: \mathbb{R}^{r} \rightarrow \mathbb{R}$ is the linear function

$$
o(s)=\sum_{i=1}^{r} s_{i}
$$

Let us observe that the canonical form (1) also includes systems of the form

$$
\left\{\begin{array}{l}
x(t+1)=\tilde{A}(p(t)) x(t) \\
p(t) \in \mathcal{P}
\end{array}\right.
$$

where $p(t) \in \mathbb{R}^{q}$ is a time-varying uncertain vector constrained into any convex bounded polytope $\mathcal{P} \subset \mathbb{R}^{q}$, and $\tilde{A}: \mathbb{R}^{q} \rightarrow \mathbb{R}^{n \times n}$ is any matrix polynomial. Indeed, one can recast (5) into (1) by expressing $p$ as a linear function of $s$, and by multiplying the monomial in $s$ by a suitable power of $o(s)$.

Problem. Establish whether (1) is robustly asymptotically stable, i.e.,

$$
\left\{\begin{array}{c}
\forall \varepsilon>0 \exists \delta>0:\|x(0)\|<\delta \Rightarrow\|x(t)\|<\varepsilon \\
\forall t \geq 0 \forall s(\cdot) \in \mathcal{S} \\
\lim _{t \rightarrow \infty} x(t)=0_{n} \forall x(0) \in \mathbb{R}^{n} \forall s(\cdot) \in \mathcal{S} .
\end{array}\right.
$$

Let us observe that the concepts of robust asymptotical stability and robust exponential stability are equivalent for the system (1) [23].

\subsection{SOS Matrix Polynomials}

A symmetric matrix homogeneous polynomial $Z: \mathbb{R}^{r} \rightarrow \mathbb{R}^{z \times z}$ is said SOS if there exist matrix homogeneous polynomials $Z_{1}, \ldots, Z_{k}: \mathbb{R}^{r} \rightarrow \mathbb{R}^{z \times z}$ such that

$$
Z(s)=\sum_{i=1}^{k} Z_{i}(s)^{\prime} Z_{i}(s) .
$$

It turns out that one can establish whether $Z(x)$ is SOS via an LMI feasibility test. Indeed, let $2 m$ be the degree of $Z(s)$ where $m$ is a nonnegative integer. Then, $Z(s)$ can be written as

$$
Z(s)=\left(b_{m}(s) \otimes I\right)^{\prime}(Y+L(\alpha))\left(b_{m}(s) \otimes I\right)
$$

where $b_{m}(s)$ is a vector of monomials of degree $m$ in $s$, which are in number equal to

$$
\sigma(r, m)=\frac{(r+m-1) !}{(r-1) ! m !}
$$


$Y \in \mathbb{R}^{z \sigma(r, m) \times z \sigma(r, m)}$ is a symmetric matrix such that

$$
Z(s)=\left(b_{m}(s) \otimes I\right)^{\prime} Y\left(b_{m}(s) \otimes I\right),
$$

$L: \mathbb{R}^{\tau(r, m, z)} \rightarrow \mathbb{R}^{z \sigma(r, m) \times z \sigma(r, m)}$ is a linear parametrization of the linear set

$$
\mathcal{L}=\left\{\tilde{L}=\tilde{L}^{\prime}: \quad\left(b_{m}(s) \otimes I\right)^{\prime} \tilde{L}\left(b_{m}(s) \otimes I\right)=0\right\}
$$

and $\alpha \in \mathbb{R}^{\tau(r, m, z)}$ is a free vector, where $\tau(r, m, z)$ is the dimension of $\mathcal{L}$ given by

$$
\begin{aligned}
\tau(r, m, z)= & \frac{1}{2} z(\sigma(r, m)(z \sigma(r, m)+1) \\
& -(z+1) \sigma(r, 2 m)) .
\end{aligned}
$$

The representation (8) is known as Gram matrix method and square matrix representation (SMR), see e.g. [24-29]. By using this representation, one has that $Z(s)$ is SOS if and only if there exists $\alpha$ satisfying the LMI

$$
Y+L(\alpha) \geq 0
$$

\section{The Gram-SOS Approach}

Let us search for a function $v: \mathbb{R}^{n} \rightarrow \mathbb{R}$ such that

$$
\forall x \in \mathbb{R}_{0}^{n} \forall s \in \mathcal{S}\left\{\begin{array}{r}
v(x)>0 \\
\Delta v(x, s)<0
\end{array}\right.
$$

where

$$
\Delta v(x, s)=v(A(s) x)-v(x) .
$$

If such a function exists, (1) is robustly asymptotically stable and $v(x)$ is a Lyapunov function for (1) for all the admissible uncertainties, see e.g. [20].

We consider a Lyapunov function candidate $v(x)$ in the class of homogeneous polynomials, i.e., a HPLF candidate. Let $2 m$ be the degree of $v(x)$. In such a case, $\Delta v(x, s)$ is a homogeneous polynomial in $x$ with degree $2 m$, and polynomial in $s$ of degree $2 d m$.

Let us observe that one can search for a HPLF candidate $v(x)$ satisfying (14) by using existing LMI techniques for optimization over polynomials. In particular, one consists of exploiting the Positivstellensatz (see e.g. [29-32]): by defining

$$
\left\{\begin{aligned}
q & =\left(s_{1}, \ldots, s_{r-1}\right)^{\prime} \\
u(q) & =\left(q^{\prime}, 1-\sum_{i=1}^{r-1} q_{i}\right)^{\prime},
\end{aligned}\right.
$$


it follows that (14) holds if there exist polynomials $f_{1}(x, q), \ldots, f_{r}(x, q)$ homogeneous with respect to $x$ such that

$$
\left.\begin{array}{c}
v(x)-\|x\|^{2 m} \\
f_{1}(x, q), \ldots, f_{r}(x, q) \\
(x, u(q))-\|x\|^{2 m}-g(x, q)
\end{array}\right\} \text { are SOS }
$$

where

$$
g(x, q)=f_{r}(x, q)+\sum_{i=1}^{r-1} q_{i}\left(f_{i}(x, q)-f_{r}(x, q)\right) .
$$

If the HPLF is quadratic (i.e., $2 m=2$ ), another possibility consists of exploiting Polya's theorem (see e.g. [29,33]): indeed, by expressing

$$
\left\{\begin{aligned}
v(x) & =x^{\prime} \tilde{V} x \\
\Delta v(x, s) & =x^{\prime}\left(A(s)^{\prime} \tilde{V} A(s)-\tilde{V}\right) x,
\end{aligned}\right.
$$

it follows that (14) holds if and only if there exists a symmetric matrix $\tilde{V} \in \mathbb{R}^{n \times n}$ and an integer $k$ such that

$$
\begin{gathered}
\tilde{V}>0 \text { and } o(s)^{k}\left(o(s)^{2 d} \tilde{V}-A(s)^{\prime} \tilde{V} A(s)\right) \text { has } \\
\text { all the matrix coefficients positive definite. }
\end{gathered}
$$

However, it can be difficult to check the LMI conditions (17) and (20) in practice. Indeed, the computational burden of the LMI condition (17) quickly grows with the degree of the HPLF candidate as it will be shown in Examples 1 and 2. Moreover, there are cases where the exponent $k$ required by the LMI condition (20) to prove robust asymptotical stability is large and failures can happen due to the computer finite precision as it will be shown in Example 3.

Let us express the HPLF candidate $v(x)$ via the Gram matrix method in Section 2.2 as

$$
v(x)=b_{m}(x)^{\prime} V b_{m}(x)
$$

where $b_{m}(x)$ has been defined in (8) and $V \in \mathbb{R}^{\sigma(n, m) \times \sigma(n, m)}$ is a symmetric matrix. Similarly, $\Delta v(x, s)$ can be expressed as

$$
\Delta v(x, s)=b_{m}(x)^{\prime} D(s) b_{m}(x)
$$

where $D: \mathbb{R}^{r} \rightarrow \mathbb{R}^{\sigma(n, m) \times \sigma(n, m)}$ is a symmetric matrix polynomial of degree $2 d m$ that can be found as follows. 
Let $J_{m}: \mathbb{R}^{n \times n} \rightarrow \mathbb{R}^{\sigma(n, m) \times \sigma(n, m)}$ be the matrix function that satisfies

$$
b_{m}(H x)=J_{m}(H) b_{m}(x)
$$

where $H \in \mathbb{R}^{n \times n}$. The matrix function $J_{m}(H)$ can be computed with the formula

$$
J_{m}(H)=\left(K_{m}^{\prime} K_{m}\right)^{-1} K_{m}^{\prime} H^{\otimes m} K_{m}
$$

where $H^{\otimes m}$ denotes the $m$-th Kronecker power of $H$, i.e.

$$
H^{\otimes m}= \begin{cases}H^{\otimes m-1} \otimes H & \text { if } m \geq 1 \\ 1 & \text { if } m=0,\end{cases}
$$

and $K_{m}$ is the matrix that satisfies

$$
x^{\otimes m}=K_{m} b_{m}(x) .
$$

It follows that

$$
D(s)=B(s)^{\prime} V B(s)-V
$$

where

$$
B(s)=J_{m}(A(s)) .
$$

Let us observe that $B(s)$ is a matrix homogeneous polynomial of degree $d m$ since $A(s)$ is a matrix homogeneous polynomial of degree $d$. Hence, $D(s)$ has degree $2 d m$.

It turns out that $D(s)$ in (27) is not the only symmetric matrix polynomial that satisfies (22). Indeed, let $L: \mathbb{R}^{\tau(n, m, 1)} \rightarrow \mathbb{R}^{\sigma(n, m) \times \sigma(n, m)}$ be a linear parametrization of $\mathcal{L}$ in (11) for $z$ replaced by 1 , and let $\beta: \mathbb{R}^{r} \rightarrow \mathbb{R}^{\tau(n, m, 1)}$ be any vector function. It follows

$$
\Delta v(x, s)=b_{m}(x)^{\prime} E(s) b_{m}(x)
$$

where

$$
E(s)=B(s)^{\prime} V B(s)-V-L(\beta(s)) .
$$

Hereafter we consider the case where $\beta(s)$ is a vector homogeneous polynomial of degree $m_{\beta}$. The following theorem provides a condition for establishing robust asymptotical stability of (1) based on the existence of suitable $V$ and $\beta(s)$.

Theorem 1 Let $m>0$ and $m_{\beta} \geq 0$ be given integers. The system (1) is robustly asymptotically stable if there exist a symmetric matrix $V \in$ 
$\mathbb{R}^{\sigma(n, m) \times \sigma(n, m)}$ and a vector homogeneous polynomial $\beta: \mathbb{R}^{r} \rightarrow \mathbb{R}^{\tau(n, m, 1)}$ of degree $m_{\beta}$ such that

$$
W(s)>0 \quad \forall s \in \mathcal{S}
$$

where $W: \mathbb{R}^{r} \rightarrow \mathbb{R}^{2 \sigma(n, m) \times 2 \sigma(n, m)}$ is the symmetric matrix homogeneous polynomial

$$
W(s)=\left(\begin{array}{cc}
o(s)^{h} V+o(s)^{h-m_{\beta}} L(\beta(s)) & o(s)^{h-d m} B(s)^{\prime} V \\
* & o(s)^{h} V
\end{array}\right)
$$

where $h$ is the degree of $W(s)$ given by

$$
h=\max \left\{d m, m_{\beta}\right\} .
$$

Proof. Let us suppose that (31) holds. Since $o(s)=1$ for all $s \in \mathcal{S},(31)$ can be rewritten as

$$
\left(\begin{array}{cc}
V+L(\beta(s)) & B(s)^{\prime} V \\
* & V
\end{array}\right)>0 .
$$

By using the Schur complement, it follows that (31) is equivalent to the condition

$$
\forall s \in \mathcal{S}\left\{\begin{aligned}
V & >0 \\
E(s) & <0 .
\end{aligned}\right.
$$

Let $x$ be any vector in $\mathbb{R}_{0}^{n}$, and let us pre- and post- multiply these inequalities by $b_{m}(x)^{\prime}$ and $b_{m}(x)$, respectively. From the first inequality we obtain that

$$
v(x)>0 \quad \forall x \in \mathbb{R}_{0}^{n} .
$$

From (23) and (28) one has that

$$
\begin{aligned}
& b_{m}(x)^{\prime} B(s)^{\prime} V B(s) b_{m}(x) \\
= & b_{m}(x)^{\prime} J(A(s))^{\prime} V J(A(s)) b_{m}(x) \\
= & b_{m}(A(s) x)^{\prime} V b_{m}(A(s) x) \\
= & v(A(s) x) .
\end{aligned}
$$

Since

$$
b_{m}(x)^{\prime} L(\beta(s)) b_{m}(x)=0,
$$

from the second inequality we obtain

$$
\Delta v(x, s)<0 \quad \forall x \in \mathbb{R}_{0}^{n} \forall s \in \mathcal{S} .
$$


Theorem 1 provides a condition for establishing whether (1) is robustly asymptotically stable. This condition is based on a novel approach that consists of three steps:

1. introducing the Gram matrices of $v(x)$ and $\Delta v(x, s)$ built with respect to $x$, i.e., $V$ and $D(s)$;

2. building the parametrization $E(s)=D(s)-L(\beta(s))$ for the Gram matrix of $\Delta v(x, s)$, where $\beta(s)$ is a free vector homogeneous polynomial;

3. defining the matrix homogeneous polynomial $W(s)$, whose positive definiteness over the simplex is equivalent to the positivity of $v(x)$ and $-\Delta v(x, s)$ for all $x \neq 0_{n}$ for all $s \in \mathcal{S}$.

Let us observe that the linear function $o(s)$ is used in the definition of $W(s)$ in order to make $W(s)$ homogeneous since $o(s)=1$ for $s \in \mathcal{S}$.

The condition provided by Theorem 1 can be checked in several ways by using LMIs. In order to describe the one adopted in this paper, let us recall the following result.

Lemma 1 ( [2]) Let $Z: \mathbb{R}^{r} \rightarrow \mathbb{R}^{z \times z}$ be a symmetric matrix homogeneous polynomial. Then,

$$
Z(s) \geq 0 \quad \forall s \in \mathcal{S}
$$

if and only if

$$
Z\left(s^{2}\right) \geq 0 \quad \forall s \in \mathbb{R}^{r}
$$

Lemma 1 states that a symmetric matrix homogeneous polynomial $Z(s)$ is positive semidefinite over the simplex if and only if $Z\left(s^{2}\right)$ is positive semidefinite everywhere. By combining Theorem 1, Lemma 1 and the definition of SOS matrix polynomial, the following result can be obtained.

Corollary 1 Let $m>0$ and $m_{\beta} \geq 0$ be given integers. The system (1) is robustly asymptotically stable if there exist a symmetric matrix $V \in$ $\mathbb{R}^{\sigma(n, m) \times \sigma(n, m)}$ and a vector homogeneous polynomial $\beta: \mathbb{R}^{r} \rightarrow \mathbb{R}^{\tau(n, m, 1)}$ of degree $m_{\beta}$ such that

$$
W\left(s^{2}\right)-\|s\|^{2 h} I \text { is } S O S .
$$


Proof. Let us suppose that (36) holds. This implies

$$
W\left(s^{2}\right)-\|s\|^{2 h} I \geq 0 \quad \forall s \in \mathbb{R}^{r}
$$

and, from Lemma 1,

$$
W(s)-o(s)^{h} I \geq 0 \quad \forall s \in \mathcal{S} .
$$

Since $o(s)=1$ for $s \in \mathcal{S}$, it follows that (31) holds, and hence from Theorem 1 we conclude that (1) is robustly asymptotically stable.

Corollary 1 provides a condition for establishing whether (1) is robustly asymptotically stable. Since this condition is based on imposing that $W\left(s^{2}\right)-$ $\|s\|^{2 h} I$ is SOS, and since $W(s)$ is built using the Gram matrices of $v(x)$ of $\Delta v(x, s)$, the overall approach is denoted as "Gram-SOS" approach. This approach is novel because it introduces the idea of generating a non-constant Gram matrix, in particular a homogeneous polynomial Gram matrix: the classic LMI variables of the SOS test in (13) (i.e., the vector $\alpha$ ) are now homogeneous polynomials (i.e., the homogeneous polynomial vector $\beta(s)$ ).

Let us observe that, since $W\left(s^{2}\right)-\|s\|^{2 h} I$ is a symmetric matrix homogeneous polynomial whose coefficients depend affine linearly on $V$ and $\beta\left(s^{2}\right)$, and since the condition that a symmetric matrix homogeneous polynomial is SOS can be rewritten as an LMI in its coefficients according to Section 2.2 , the condition provided by Corollary 1 is an LMI feasibility test.

The LMI condition provided by Corollary 1 is sufficient for any chosen integers $m$ and $m_{\beta}$, which define the degree of the HPLF candidate $v(x)$ (equal to $2 \mathrm{~m}$ ) and of the vector homogeneous polynomial $\beta(s)$ (equal to $\left.m_{\beta}\right)$.

\section{Conservatism Analysis}

It is useful to clarify whether the conditions provided by Theorem 1 and Corollary 1 cover the case of quadratic robust stability. In particular, (1) is said quadratically robustly asymptotically stable if (14) holds with a quadratic function $v(x)$. By expressing such a $v(x)$ and the corresponding $\Delta v(x, s)$ as in (19) for a symmetric matrix $\tilde{V} \in \mathbb{R}^{n \times n}$, it follows that (14) is equivalent to the condition

$$
\forall s \in \mathcal{S}\left\{\begin{aligned}
\tilde{V} & >0 \\
A(s)^{\prime} \tilde{V} A(s)-\tilde{V} & <0
\end{aligned}\right.
$$


Let us observe that the HPLF candidate $v(x)$ in (21) is a quadratic function whenever $m=1$. In such a case, the vector homogeneous polynomial $\beta(s)$ is not needed in the condition provided by Theorem 1 because, for $m=1$, one has

$$
\mathcal{L}=\emptyset
$$

and, hence, $L(\beta(s))=0$. This means that, for $m=1, W(s)$ does not depend on $\beta(s)$ and the degree of $W(s)$ is $h=d$.

The next result states that the condition provided by Theorem 1 with $m=1$ is sufficient and necessary for quadratic robust asymptotic stability, and that this is equivalent to the LMI condition provided by Corollary 1 if $d=1$.

Theorem 2 The system (1) is quadratically robustly asymptotically stable if and only if the condition provided by Theorem 1 holds with $m=1$. Moreover, if $d=1$, this is equivalent to the fulfillment of the LMI condition provided by Corollary 1 with $m=1$.

Proof. The fact that (1) is quadratically robustly asymptotically stable if and only if the condition provided by Theorem 1 holds with $m=1$ directly follows by observing that (37) is equivalent to (31) by using the Schur complement since $o(s)=1$ for $s \in \mathcal{S}$ and $L(\beta(s))=0$. Hence, let us prove that, if $d=1,(37)$ is equivalent to (36).

" $\Rightarrow$ " Suppose that (1) is quadratically robustly asymptotically stable. Hence, there exists $\tilde{V}$ satisfying (37). Since $o(s)=1$ for $s \in \mathcal{S}$, this implies that

$$
\left(\begin{array}{cc}
o(s) \tilde{V} & A(s)^{\prime} \tilde{V} \\
* & o(s) \tilde{V}
\end{array}\right)>0 \quad \forall s \in \mathcal{S} .
$$

Let us observe that the left hand side of the previous condition coincides with $W(s)$ for $m=1$ by replacing $\tilde{V}$ with $V$ since, in such a case, one has that

$$
\left\{\begin{aligned}
B(s) & =A(s) \\
L(\beta(s)) & =0
\end{aligned}\right.
$$

Since $W(s)$ is linear in $s$ in this case, it follows that $W(s)$ can be expressed as

$$
W(s)=\sum_{i=1}^{r} s_{i} W_{i}
$$

for some symmetric matrices $W_{i}, \ldots, W_{r}$. Hence, the positive definiteness of $W(s)$ over $\mathcal{S}$ is equivalent to

$$
W_{i}>0 \quad \forall i=1, \ldots, r .
$$


This implies that $\lambda_{1}>0$ where

$$
\lambda_{1}=\min _{i=1, \ldots, r} \lambda_{\min }\left(W_{i}\right) .
$$

Since $W_{i}-\lambda_{1} I$ is positive semidefinite for all $i=1, \ldots, r$, one can write

$$
W_{i}-\lambda_{1} I_{2 a}=\bar{W}_{i}^{\prime} \bar{W}_{i} \quad \forall i=1, \ldots, r
$$

where $\bar{W}_{i}$ is the Cholesky factorization of $W_{i}-\lambda_{1} I$. It follows that

$$
\begin{aligned}
W\left(s^{2}\right)-\lambda_{1}\|s\|^{2} I & =\sum_{i=1}^{r} s_{i}^{2} W_{i}-\lambda_{1}\|s\|^{2} I \\
& =\sum_{i=1}^{r} s_{i}^{2}\left(W_{i}-\lambda_{1} I\right) \\
& =\sum_{i=1}^{r}\left(s_{i} \bar{W}_{i}\right)^{\prime}\left(s_{i} \bar{W}_{i}\right)
\end{aligned}
$$

which means that

$$
W\left(s^{2}\right)-\lambda_{1}\|s\|^{2} I \text { is SOS. }
$$

Since $W\left(s^{2}\right)$ and $\lambda_{1}$ are linear in $V$, we conclude that (36) holds by replacing $V$ as

$$
V \rightarrow \lambda_{2} V \quad \forall \lambda_{2} \in\left[\frac{1}{\lambda_{1}}, \infty\right)
$$

" $\Leftarrow$ " Suppose that there exists $V$ satisfying the LMI condition (36) with $m=1$. This implies

$$
W\left(s^{2}\right)-\|s\|^{2} I \geq 0 \quad \forall s \in \mathbb{R}^{r} .
$$

From Lemma 1 it follows that

$$
W(s)-I \geq 0 \quad \forall s \in \mathcal{S}
$$

which implies (37) by replacing $V$ with $\tilde{V}$ since $B(s)=A(s)$ in this case.

The next result investigates the conservatism of the LMI condition provided by Corollary 1, clarifying that this condition is not only sufficient but also necessary for sufficiently large degrees of the HPLF candidate $v(x)$ and of the vector homogeneous polynomial $\beta(s)$.

Theorem 3 Suppose that (1) is robustly asymptotically stable. Then, there exist sufficiently large integers $m$ and $m_{\beta}$ such that the LMI condition provided by Corollary 1 is satisfied. 
Proof. Since (1) is robustly asymptotically stable, from Section VI of [20] it follows that there exists a polyhedral Lyapunov function, which can be expressed as $\|C x\|_{\infty}$ for some full rank matrix $C$. From Theorem 3.2 of [11] this implies that $\|C x\|_{2 p}$ is a Lyapunov function for some positive integer $p$. Indeed, as clarified in [11], the existence of the latter Lyapunov function is an expected consequence of the fact that $\|C x\|_{2 p}$ uniformly converges to $\|C x\|_{\infty}$ on every compact set as $p$ tends to $\infty$. Since $\|C x\|_{2 p}$ is a Lyapunov function, it follows that also its $2 p$-th power

$$
v(x)=\|C x\|_{2 p}^{2 p}
$$

is, i.e., (14) holds with $v(x)$ homogeneous polynomial of degree $2 p$. Let $k$ be a positive integer, and define

$$
\bar{v}(x)=v(x)^{k} .
$$

It follows that $\bar{v}(x)$ is a homogeneous polynomial of degree $2 \bar{m}$ where

$$
\bar{m}=k p .
$$

Let us observe that $v(x)$ is a SOS polynomial being the $2 p$-th power of the $2 p$-norm of a linear function. This implies that also $\bar{v}(x)$ is a SOS polynomial since it is a power of a SOS polynomial. Since $C$ has full rank, it follows that $\bar{v}(x)$ is positive definite, in particular it admits a positive definite Gram matrix $\bar{V}$. Let us consider the time difference of $\bar{v}(x)$. This is given by

$$
\begin{aligned}
\Delta \bar{v}(x, s) & =v(A(s) x)^{k}-v(x)^{k} \\
& =g(x, s) \Delta v(x, s)
\end{aligned}
$$

where

$$
g(x, s)=\sum_{i=0}^{k-1} v(A(s) x)^{k-1-i} v(x)^{i} .
$$

Since (14) holds, it follows that

$$
g(x, s)>0 \quad \forall x \in \mathbb{R}_{0}^{n} \forall s \in \mathcal{S}
$$

and, hence,

$$
-\Delta \bar{v}(x, s)>0 \quad \forall x \in \mathbb{R}_{0}^{n} \forall s \in \mathcal{S} .
$$

Moreover, there exists a sufficiently large $k$ such that $-\Delta \bar{v}(x, s)$ is SOS for all $s \in \mathcal{S}$. In fact, for all $s \in \mathcal{S},-\Delta v(x, s)$ is a positive definite homogeneous polynomial in $x$ and $g(x, s)$ is the sum of terms of the form 
$v(A(s) x)^{k-1-i} v(x)^{i}$. Since both $v(A(s) x)$ and $v(x)$ are SOS positive definite homogeneous polynomials in $x$ for all $s \in \mathcal{S}$, from Section 2.1.8 of [34] it follows that there exist finite integers $k_{1}(s)$ and $k_{2}(s)$ such that $-v(A(s) x)^{k_{1}(s)} \Delta v(x, s)$ and $-v(x)^{k_{2}(s)} \Delta v(x, s)$ are SOS for all $s \in \mathcal{S}$. Hence, one can ensure that $-v(A(s) x)^{k-1-i} v(x)^{i} \Delta v(x, s)$ is SOS for all $i=0, \ldots, k-$ 1 for all $s \in \mathcal{S}$ by choosing

$$
k=\max _{s \in \mathcal{S}} 2 \max \left\{k_{1}(s), k_{2}(s)\right\}+2
$$

which does exist since $k_{1}(s)$ and $k_{2}(s)$ are bounded and $\mathcal{S}$ is compact. Therefore, $-\Delta \bar{v}(x, s)$ is SOS for all $s \in \mathcal{S}$, which implies that $\Delta \bar{v}(x, s)$ admits a Gram matrix $\bar{E}(s)$ satisfying

$$
\bar{E}(s)<0 \quad \forall s \in \mathcal{S} .
$$

From (29) it follows that $\bar{E}(s)$ can be expressed as

$$
\bar{E}(s)=B(s)^{\prime} \bar{V} B(s)-\bar{V}-\bar{L}(\theta(s))
$$

for some function $\theta(s)$, where $\bar{L}=\bar{L}^{\prime}: \mathbb{R}^{\tau(n, \bar{m}, 1)} \rightarrow \mathbb{R}^{\sigma(n, \bar{m}) \times \sigma(n, \bar{m})}$ is a linear parametrization of $\mathcal{L}$ in (11) for $m$ and $z$ replaced by $\bar{m}$ and 1 , respectively. This implies that

$$
\hat{W}(s)>0 \quad \forall s \in \mathcal{S}
$$

where

$$
\hat{W}(s)=\left(\begin{array}{cc}
\bar{V}+\bar{L}(\theta(s)) & \bar{V} B(s)^{\prime} \\
* & \bar{V}
\end{array}\right) .
$$

Since $\mathcal{S}$ is compact, there exist a sufficiently large integer $k$ and a vector homogeneous polynomial $\bar{\beta}(s)$ of degree $\bar{m}_{\beta}$ that approximates arbitrary well $\theta(s)$ over $\mathcal{S}$. Observe that $\bar{\beta}(s)$ can be assumed homogeneous without loss of generality since each monomial can be multiplied by a suitable power of $o(s)$ due to the fact that $o(s)=1$ for $s \in \mathcal{S}$. This means that there exist a sufficiently large integer $k$ and a homogeneous vector polynomial $\bar{\beta}(s)$ of degree $\bar{m}_{\beta}$ such that

$$
\bar{W}(s)>0 \quad \forall s \in \mathcal{S}
$$

where

$$
\bar{W}(s)=\left(\begin{array}{cc}
o(s)^{\bar{h}} \bar{V}+o(s)^{\bar{h}-\bar{m}_{\beta}} \bar{L}(\bar{\beta}(s)) & o(s)^{\bar{h}-d \bar{m}} B(s)^{\prime} \bar{V} \\
* & o(s)^{\bar{h}} \bar{V}
\end{array}\right)
$$

and

$$
\bar{h}=\max \left\{d \bar{m}, \bar{m}_{\beta}\right\} .
$$


Let us observe that $\bar{W}(s)$ is the matrix $W(s)$ in (32) built for $m, V$ and $\beta(s)$ replaced, respectively, by $\bar{m}, \bar{V}$ and $\bar{\beta}(s)$.

Since $\bar{W}(s)$ is a symmetric matrix homogeneous polynomial, it follows that there exists a nonnegative integer $l$ such that the matrix coefficients of $o(s)^{l} \bar{W}(s)$ are positive definite due to Polya's theorem, see e.g. [29,33]. This implies that the matrix coefficients of $\bar{W}(s)$ are positive definite by replacing $\bar{m}$ with $\bar{m}+l$. Indeed, with this replacement one gets $o(s)^{\bar{h}}, o(s)^{\bar{h}-\bar{m}_{\beta}}$ and $o(s)^{d \bar{m}}$ replaced by $o(s)^{\bar{h}+l}, o(s)^{\bar{h}-\bar{m}_{\beta}+l}$ and $o(s)^{d \bar{m}+l}$, respectively, and, hence, $\bar{W}(s)$ replaced by $o(s)^{l} \bar{W}(s)$. Consequently, $\bar{W}(s)$ can be expressed as

$$
\bar{W}(s)=\sum_{\substack{i_{1}+\ldots+i_{r}=\bar{m}+l \\ i_{1} \geq 0, \ldots, i_{r} \geq 0}} \bar{W}_{i_{1}, \cdots, i_{r}} \prod_{j=1}^{r} s_{j}^{i_{j}}
$$

with $\bar{W}_{i_{1} \cdots i_{r}}>0$. This means that $\bar{W}\left(s^{2}\right)$ is SOS since

$$
\bar{W}\left(s^{2}\right)=\sum_{\substack{i_{1}+\ldots+i_{r}=\bar{m}+l \\ i_{1} \geq 0, \ldots, i_{r} \geq 0}} C_{i_{1}, \cdots, i_{r}}(s)^{\prime} C_{i_{1}, \cdots, i_{r}}(s)
$$

where

$$
C_{i_{1}, \cdots, i_{r}}(s)=D_{i_{1}, \cdots, i_{r}} \prod_{j=1}^{r} s_{j}^{i_{j}}
$$

and $D_{i_{1}, \cdots, i_{r}}$ satisfies the Cholesky factorization

$$
\bar{W}_{i_{1}, \cdots, i_{r}}=D_{i_{1}, \cdots, i_{r}}^{\prime} D_{i_{1}, \cdots, i_{r}} .
$$

Therefore, (36) holds by choosing $m=\bar{m}$ and $m_{\beta}=\bar{m}_{\beta}+l$.

\section{Complexity Reduction}

The number of LMI scalar variables in the LMI condition provided by Corollary 1 is given by the sum of the number of the non-symmetric entries of $V$, the number of the coefficients of the vector homogeneous polynomial $\beta(s)$, and the length of the vector $\alpha$ in the representation (8). Considering for brevity the case $m_{\beta}=d m$, the number of LMI scalar variables turns out to be

$$
\begin{aligned}
\eta= & \frac{1}{2} \sigma(n, m)(\sigma(n, m)+1)+\sigma(r, m) \tau(n, m, 1) \\
& +\tau(r, d m, 2 \sigma(n, m)) .
\end{aligned}
$$


Table 1a shows this number in the case $r=2$ and $d=1$ for some values of $n$ and $m$.

It turns out that the length of the vector $\alpha$ is the dominant term in $\eta$. Fortunately, this can be significantly reduced in the case of the LMI condition provided by Corollary 1 due to the structure of $W\left(s^{2}\right)-\|s\|^{2 h} I$.

Indeed, let $Z: \mathbb{R}^{r} \rightarrow \mathbb{R}^{z \times z}$ be a symmetric matrix homogeneous polynomial of degree $2 m$ with even powers only, i.e., such that

$$
Z(s)=\bar{Z}\left(s^{2}\right)
$$

for some symmetric matrix homogeneous polynomial $\bar{Z}: \mathbb{R}^{r} \rightarrow \mathbb{R}^{z \times z}$ of degree $m$. Let us define the set

$$
\begin{aligned}
& \mathcal{N}=\left\{N=N^{\prime}: \quad\left(b_{m}(s) \otimes I\right)^{\prime} N\right. \\
& \cdot\left(b_{m}(s) \otimes I\right)=0 \text { and } N_{i j}=0 \quad \forall i, j: \\
& \left.\left(b_{m}(s)\right)_{i}\left(b_{m}(s)\right)_{j} \text { has at least an odd power }\right\}
\end{aligned}
$$

where $N_{i j} \in \mathbb{R}^{z \times z}$ is the $(i, j)$-block of $N=N^{\prime} \in \mathbb{R}^{z \sigma(r, m) \times z \sigma(r, m)}$, i.e.

$$
N=\left(\begin{array}{ccc}
N_{11} & \ldots & N_{1 c} \\
\vdots & \ddots & \vdots \\
\star & \ldots & N_{c c}
\end{array}\right)
$$

Lastly, let $\xi(r, m)$ be the number of distinct pairs of $r$-tuples of nonnegative integers with sum equal to $m$ such that the sum of the two $r$-tuples in the pair has only even integers. It can be verified that

$$
\begin{aligned}
\xi(r, m)= & \sum_{i=0}^{\min \left\{\left\lfloor\frac{r-\omega}{2}\right\rfloor\left\lfloor\left\lfloor\frac{m}{2}\right\rfloor\right\}\right.} \frac{1}{2} \sigma(r-2 i-\omega+1,2 i+\omega) \\
& \cdot \sigma\left(r,\left\lfloor\frac{m}{2}\right\rfloor-i\right)\left(\sigma\left(r,\left\lfloor\frac{m}{2}\right\rfloor-i\right)+1\right)
\end{aligned}
$$

where

$$
\omega= \begin{cases}0 & \text { if } m \text { is even } \\ 1 & \text { otherwise }\end{cases}
$$

Theorem 4 Let $Z: \mathbb{R}^{r} \rightarrow \mathbb{R}^{z \times z}$ be a symmetric matrix homogeneous polynomial of degree $2 m$ with even powers only. Let $Y \in \mathbb{R}^{z \sigma(r, m) \times z \sigma(r, m)}$ be a symmetric matrix satisfying (10). Let $N: \mathbb{R}^{\phi(r, m, z)} \rightarrow \mathbb{R}^{z \sigma(r, m) \times z \sigma(r, m)}$ be a 
linear parametrization of the linear set $\mathcal{N}$ where $\phi(r, m, z)$ is the dimension of $\mathcal{N}$ given by

$$
\phi(r, m, z)=(\xi(r, m)-\sigma(r, m)) z^{2} .
$$

Then,

$$
Z\left(s^{2}\right) \text { is } S O S
$$

if and only if there exists $\gamma \in \mathbb{R}^{\phi(r, m, z)}$ such that

$$
Y+N(\gamma) \geq 0
$$

Proof. First, let us observe that $\mathcal{N}$ is a linear set. Indeed, for all $N_{1}, N_{2} \in \mathcal{N}$ and for all $y_{1}, y_{2} \in \mathbb{R}$, one has that

$$
N=\sum_{k=1}^{2} y_{k} N_{k}
$$

belongs to $\mathcal{N}$ since

$$
\begin{aligned}
& \left(b_{m}(s) \otimes I\right)^{\prime} N\left(b_{m}(s) \otimes I\right) \\
= & \sum_{k=1}^{2} y_{k}\left(b_{m}(s) \otimes I\right)^{\prime} N_{k}\left(b_{m}(s) \otimes I\right) \\
= & 0
\end{aligned}
$$

and

$$
N_{i j}=\sum_{k=1}^{2} y_{k} N_{k i j}=0
$$

for all $i, j$ such that $\left(b_{m}(s)\right)_{i}\left(b_{m}(s)\right)_{j}$ has at least an odd power, where $N_{k i j} \in \mathbb{R}^{z \times z}$ is the $(i, j)$-block of $N_{k}$.

Second, let us observe that $\phi(r, m, z)$ is the dimension of $\mathcal{N}$ because such a dimension is given by $d_{1}-d_{2}$ where $d_{1}$ is the number of non-symmetric entries in a symmetric matrix $N \in \mathbb{R}^{z \sigma(r, m) \times z \sigma(r, m)}$ such that $N_{i j}=0$ for all $i, j$ such that $\left(b_{m}(s)\right)_{i}\left(b_{m}(s)\right)_{j}$ has at least an odd power, given by

$$
d_{1}=(\xi(r, m)-\sigma(r, m)) z^{2}+\frac{1}{2} z(z+1) \sigma(r, m),
$$

and $d_{2}$ is the number of constraints imposed by the condition $\left(b_{m}(s) \otimes I\right)^{\prime} N$ $\cdot\left(b_{m}(s) \otimes I\right)=0$, given by

$$
d_{2}=\frac{1}{2} z(z+1) \sigma(r, m) .
$$


Third, let us suppose that there exists $\gamma \in \mathbb{R}^{\phi(r, m, z)}$ such that (47) holds. Hence, there exists a Cholesky factor of $Y+N(\gamma)$ that we denote as $X$, i.e.,

$$
Y+N(\gamma)=X^{\prime} X
$$

It follows that

$$
\begin{aligned}
Z(s) & =\left(b_{m}(s) \otimes I\right)^{\prime}(Y+N(\gamma))\left(b_{m}(s) \otimes I\right) \\
& =\left(b_{m}(s) \otimes I\right)^{\prime} X^{\prime} X\left(b_{m}(s) \otimes I\right) .
\end{aligned}
$$

Let us partition $X$ according to

$$
X=\left(X_{1}^{\prime}, \ldots, X_{c}^{\prime}\right)^{\prime}
$$

where $X_{i} \in \mathbb{R}^{z \times z \sigma(r, m)}, i=1, \ldots, \sigma(r, m)$. It follows that

$$
Z\left(s^{2}\right)=\sum_{i=1}^{\sigma(r, m)} Z_{i}(s)^{\prime} Z_{i}(s)
$$

where

$$
Z_{i}(s)=X_{i}\left(b(s) \otimes I_{z}\right)
$$

i.e. (46) holds.

Lastly, let us suppose that (46) holds. Then, from Section 2.2, it follows that there exists $\alpha$ such that (13) holds. Let us observe that

$$
\mathcal{N} \subseteq \mathcal{L}
$$

There are two cases: $L(\alpha) \in \mathcal{N}$ or $L(\alpha) \notin \mathcal{N}$. In the former case, it directly follows that there exists $\gamma$ such that

$$
N(\gamma)=L(\alpha)
$$

and, hence, (47) holds. In the latter case, let $\gamma$ be such that $N(\gamma)$ is the projection of $L(\alpha)$ onto $\mathcal{N}$, i.e.

$$
L(\alpha)=N(\gamma)+N_{0}
$$

where $N_{0} \in \mathbb{R}^{z \sigma(r, m) \times z \sigma(r, m)}$ is a symmetric matrix whose $(i, j)$-block $N_{0 i j}$ is zero for all $i, j$ such that $\left(b_{m}(s)\right)_{i}\left(b_{m}(s)\right)_{j}$ has only even powers. Since the diagonal blocks of $N_{0}$ are null, and since the possible non-zero blocks of $N_{0}$ are null in $Y+N(\gamma)$, it follows that

$$
\mu_{i}\left(Y+N(\gamma)+N_{0}\right) \leq \mu_{i}(Y+N(\gamma))
$$


for all $i=1, \ldots, z \sigma(r, m)$, where $\mu_{i}(N)$ denotes the $i$-th principal minor of $N$. This implies that (47) holds since $Y+N(\gamma)+N_{0} \geq 0$.

Theorem 4 provides an alternative LMI feasibility test for establishing whether a symmetric matrix homogeneous polynomial with even powers only is SOS. This test is sufficient and necessary, and, with respect to the existing test in Section 2.2, has the advantage of a significantly smaller computational burden. Indeed, by using this test for checking the LMI condition provided by Corollary 1, one has that the number of LMI scalar variables in (36) is

$$
\begin{aligned}
\eta^{*}= & \frac{1}{2} \sigma(n, m)(\sigma(n, m)+1)+\sigma(r, m) \tau(n, m, 1) \\
& +\phi(r, d m, 2 \sigma(n, m)) .
\end{aligned}
$$

Table $1 \mathrm{~b}$ shows this number in the case $r=2$ and $d=1$ for some values of $n$ and $m$. As we can see, $\eta^{*}$ is significantly smaller than $\eta$.

\begin{tabular}{c|ccc}
$\eta$ & $m=1$ & $m=2$ & $m=3$ \\
\hline$n=1$ & 2 & 7 & 16 \\
$n=2$ & 9 & 75 & 298 \\
$n=3$ & 21 & 315 & 1933 \\
$n=4$ & 38 & 895 & 7854
\end{tabular}

(a)

\begin{tabular}{c|ccc}
$\eta^{*}$ & $m=1$ & $m=2$ & $m=3$ \\
\hline$n=1$ & 1 & 5 & 9 \\
$n=2$ & 3 & 45 & 150 \\
$n=3$ & 6 & 183 & 963 \\
$n=4$ & 10 & 515 & 3914
\end{tabular}

(b)

Table 1: Number of LMI scalar variables $\eta$ (a) and $\eta^{*}$ (b) in the case $r=2$ and $d=1$ for some values of $n$ and $m$.

\section{$6 \quad$ Illustrative Examples}

In this section we present some examples that illustrate the use of the proposed approach. The computations have been done in Matlab using the toolbox SeDuMi [35]. The reported HPLFs are normalized under the con- 
straint trace $(V)=10$. The LMI condition provided by Corollary 1 is used with the choice $m_{\beta}=m$.

For comparison, we also investigate the alternative LMI conditions (17) based on the Positivstellensatz, (20) based on Polya's theorem, and [21]. We denote with $\eta_{\text {Pos }}$ the number of LMI scalar variables in (17), which is the number of decision variables plus the number of non-symmetric entries in the Gram matrices (reduced by using the Newton polytope) less the number of monomials that can be generated by such Gram matrices.

\subsection{Example 1}

Let us consider

$$
x(t+1)=\left(\begin{array}{cc}
0 & 1 \\
-0.8 & p(t)
\end{array}\right) x(t)
$$

where $x(t) \in \mathbb{R}^{2}$ is the state and $p(t) \in \mathbb{R}$ is the time-varying uncertainty confined into the interval

$$
\mathcal{P}=[0, \zeta] .
$$

The problem consists of determining the largest value of $\zeta$, denoted by $\zeta^{*}$, such that the system is robustly asymptotically stable for all time-varying $p(t)$ in $\mathcal{P}$.

This system is in the form (5), and can be rewritten in the canonical form (1) with $r=2$ and $p=\zeta s_{2}$, which provides

$$
A(s)=\left(\begin{array}{cc}
0 & o(s) \\
-0.8 o(s) & \zeta s_{2}
\end{array}\right) .
$$

In order to estimate $\zeta^{*}$, we use a bisection algorithm where the LMI condition provided by Corollary 1 is checked at each step. For any chosen value of $m$ this will provide a lower bound of $\zeta^{*}$.

For $m=1$ we have $B(s)=A(s)$ and we find the lower bound $\zeta_{1}=0.397$ of $\zeta^{*}$. The HPLF ensuring $\zeta_{1}$ is given by

$$
v(x)=4.444 x_{1}^{2}-1.104 x_{2} x_{1}+5.556 x_{2}^{2} .
$$

Figure 1 shows a level set of $v(x)$ (inner curve). Let us observe that the vector homogeneous polynomial $\beta(s)$ is not needed for $m=1$ since $\mathcal{L}$ in (11) is empty in this case and, hence, $L(\beta(s))=0$. The number of LMI scalar variables in (36) is $\eta^{*}=3$ (the average computational time is $0.1 \mathrm{~s}$ ).

This lower bound can also be found by using (17) with polynomials $f_{i}(x, q)$ of degree not smaller than 2 , for which the number of LMI scalar variables is $\eta_{\text {Pos }}=24$. 
For $m=2$ we have

$$
B(s)=\left(\begin{array}{ccc}
0 & 0 & o(s)^{2} \\
0 & -0.8 o(s)^{2} & \zeta\left(s_{1} s_{2}+s_{2}^{2}\right) \\
0.64 o(s)^{2} & -1.6 \zeta s_{2} o(s) & \zeta^{2} s_{2}^{2}
\end{array}\right)
$$

and we find the lower bound $\zeta_{2}=0.471$. The HPLF ensuring $\zeta_{2}$ is given by

$$
\begin{aligned}
v(x)= & 2.619 x_{1}^{4}+1.198 x_{1}^{3} x_{2}+6.907 x_{2}^{2} x_{1}^{2}-4.788 x_{2}^{3} x_{1} \\
& +4.442 x_{2}^{4} .
\end{aligned}
$$

Figure 1 shows a level set of $v(x)$ (central curve). The number of LMI scalar variables in (36) is $\eta^{*}=45$ (the average computational time is $0.4 \mathrm{~s}$ ).

This lower bound can also be found by using (17) with polynomials $f_{i}(x, q)$ of degree not smaller than 4 , for which the number of LMI scalar variables is $\eta_{\text {Pos }}=116$.

For $m=3$ we find the lower bound $\zeta_{3}=0.523$. The HPLF ensuring $\zeta_{3}$ is given by

$$
\begin{aligned}
v(x)= & 0.982 x_{1}^{6}+0.632 x_{1}^{5} x_{2}+4.162 x_{1}^{4} x_{2}^{2}+0.142 x_{2}^{3} x_{1}^{3} \\
& +6.457 x_{2}^{4} x_{1}^{2}-6.059 x_{2}^{5} x_{1}+2.802 x_{2}^{6} .
\end{aligned}
$$

Figure 1 shows a level set of $v(x)$ (outer curve). The number of LMI scalar variables in (36) is $\eta^{*}=150$ (the average computational time is $1.4 \mathrm{~s}$ ).

This lower bound can also be found by using (17) with polynomials $f_{i}(x, q)$ of degree not smaller than 6 , for which the number of LMI scalar variables is $\eta_{\text {Pos }}=369$.

For comparison, we also consider the condition proposed in [21], which provides the lower bound $\zeta_{D B}=0.438$.

\subsection{Example 2}

Let us consider the problem of establishing robust asymptotical stability of

$$
x(t+1)=\left(\begin{array}{ccc}
0 & -0.5 & 0.5+0.4 p_{2}(t) \\
0.5 p_{1}(t) & 0 & 0.4 \\
-0.8 & 0.4 p_{2}(t) & -0.3 p_{1}(t)
\end{array}\right) x(t)
$$

where $x(t) \in \mathbb{R}^{3}$ is the state and $p(t) \in \mathbb{R}^{2}$ is the time-varying uncertainty confined into the polytope

$$
\mathcal{P}=\operatorname{conv}\left\{\left(\begin{array}{l}
-1 \\
-1
\end{array}\right),\left(\begin{array}{c}
1 \\
-1
\end{array}\right),\left(\begin{array}{l}
0 \\
1
\end{array}\right)\right\} .
$$




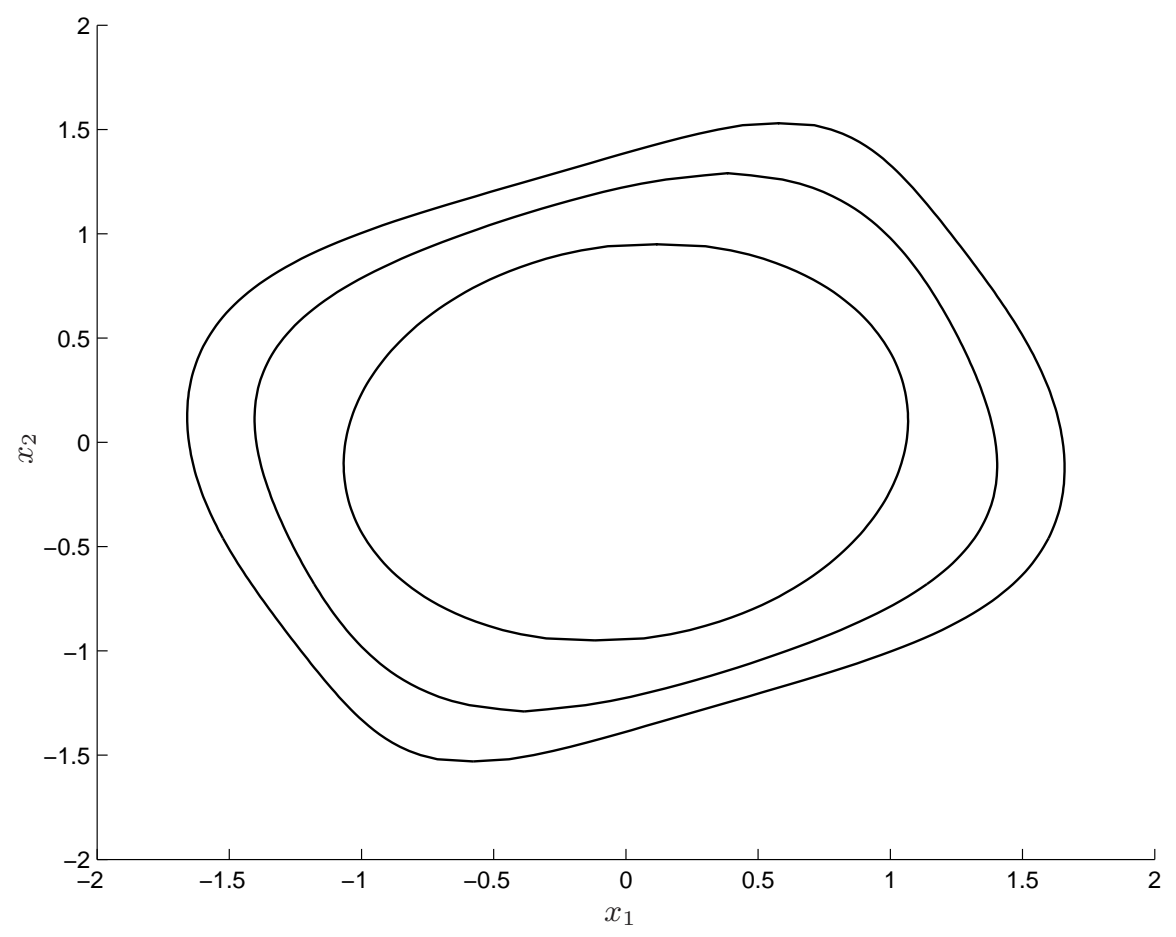

Figure 1: Example 1: a level set of the HPLF found for $m=1$ (inner curve), $m=2$ (central curve), and $m=3$ (outer curve).

This system is in the form (5), and can be rewritten in the canonical form (1) with $r=3$ and $p$ given by

$$
p=\left(\begin{array}{ccc}
-1 & 1 & 0 \\
-1 & -1 & 1
\end{array}\right) s .
$$

For $m=1$ we find that the LMI condition provided by Corollary 1 is not satisfied. Hence, we increase $m$, and for $m=2$ we find that this condition holds, hence implying that the system is robustly asymptotically stable. Figure 2 shows a level set of the found HPLF $v(x)$. The number of LMI scalar variables in (36) is $\eta^{*}=489$ (the computational time is $2.5 \mathrm{~s}$ ).

This HPLF can also be found by using (17) with polynomials $f_{i}(x, q)$ of degree not smaller than 4 , for which the number of LMI scalar variables is $\eta_{\text {Pos }}=2460$.

For comparison, we also consider the condition proposed in [21], which however is not satisfied in this case. 


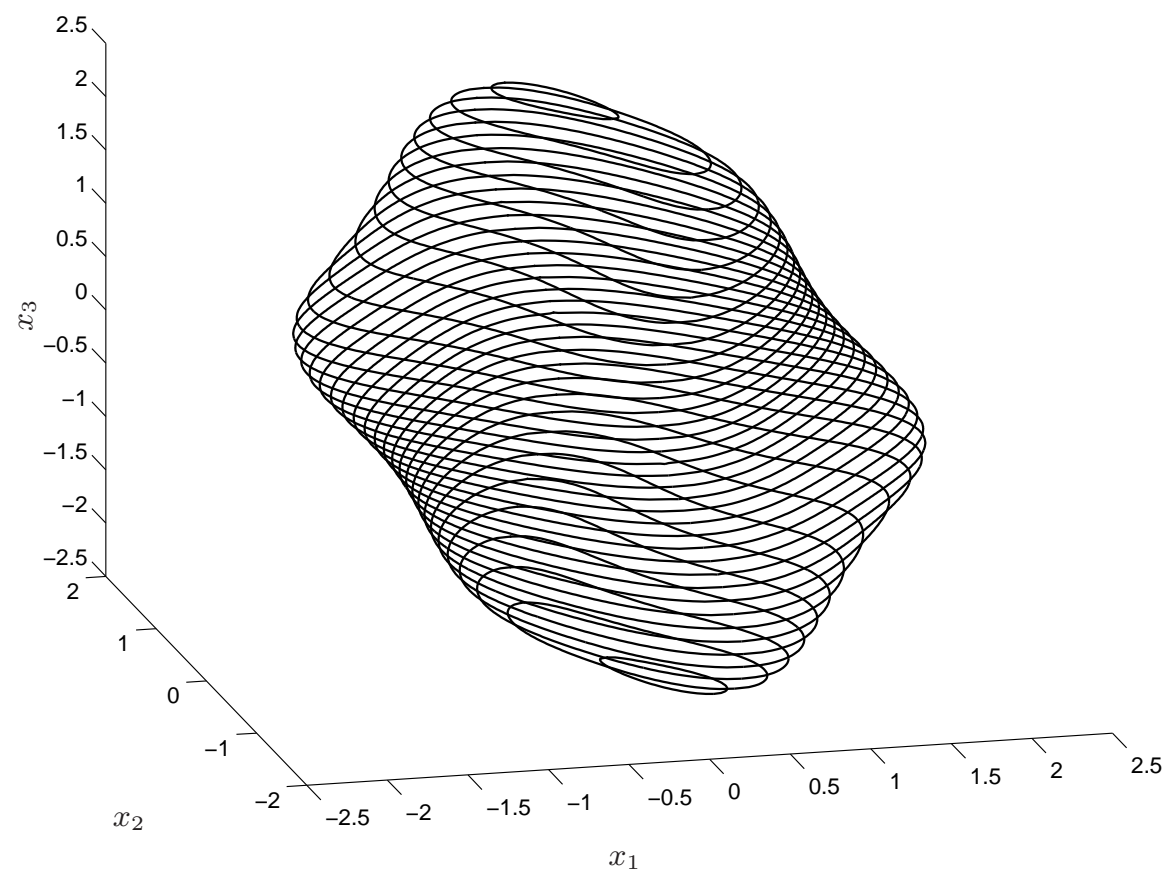

Figure 2: Example 2: a level set of the HPLF $v(x)$ found for $m=2$.

\subsection{Example 3}

We conclude this section with a simple example in order to investigate the alternative LMI condition (20) based on Polya's theorem. Let us consider

$$
x(t+1)=3.98 p(t)(1-p(t)) x(t)
$$

where $x(t) \in \mathbb{R}$ is the state and $p(t) \in \mathbb{R}$ is the time-varying uncertainty confined into the interval

$$
\mathcal{P}=[0,1] .
$$

This system is in the form (5), and can be rewritten in the canonical form (1) with $r=2$ and $p=s_{2}$, which provides

$$
A(s)=3.98 s_{1} s_{2} \text {. }
$$

The system is robustly asymptotically stable since the state is scalar and $A(s)$ ranges in $[0,0.995]$ for $s \in \mathcal{S}$. 
This can be proved with a quadratic Lyapunov function. Indeed, for $m=1$ we find that the LMI condition provided by Corollary 1 is satisfied. The number of LMI scalar variables in (36) is $\eta^{*}=5$ (the computational time is $0.1 \mathrm{~s}$ ).

However, we are unable to prove this by using the alternative LMI condition (20) based on Polya's theorem. Indeed, the value of the exponent $k$ required in (20) to prove robust asymptotical stability is 197, but for such a value our Matlab implementation of (20) crashes due to the computer finite precision (the coefficients of $o(s)^{k}$ range from 1 to $1.138 \cdot 10^{58}$ for $k=197$ ).

\section{Conclusions}

This paper has proposed a novel approach, referred to as the Gram-SOS approach, for establishing robust asymptotical stability of discrete-time systems polynomially affected by time-varying uncertainty confined into a polytope. It has been shown that the proposed approach is sufficient for any degree of the HPLF candidate, that includes quadratic robust stability as a special case, and that is also necessary for a sufficiently large degree of the HPLF candidate.

The computational burden of the proposed approach quickly grows with the dimension of the system, however this seems unavoidable in order to achieve nonconservatism. Moreover, it is expected that the fast increasing power of nowadays computers can soon allow one to consider also non-small scale systems. Also, one can always freeze some LMI variables in the proposed approach in order to fit the available computer power (clearly, at the expenses of some conservatism).

\section{Acknowledgement}

The author would like to thank the Associate Editor and the Reviewers for their useful comments. He also thanks Franco Blanchini for his help in writing the proof of Theorem 3. This work is supported in part by the Research Grants Council of Hong Kong under Grant HKU711213E.

\section{References}

[1] B. R. Barmish. New Tools for Robustness of Linear Systems. Mcmillan Publishing Company, New York, 1994. 
[2] G. Chesi, A. Garulli, A. Tesi, and A. Vicino. Homogeneous Polynomial Forms for Robustness Analysis of Uncertain Systems. Springer, 2009.

[3] I. R. Petersen and R. Tempo. Robust control of uncertain systems: Classical results and recent developments. Automatica, 50(5):13151335, 2013.

[4] S. Boyd, L. El Ghaoui, E. Feron, and V. Balakrishnan. Linear Matrix Inequalities in System and Control Theory. SIAM, 1994.

[5] L. Xie, S. Shishkin, and M. Fu. Piecewise Lyapunov functions for robust stability of linear time-varying systems. Systems and Control Letters, 31:165-171, 1997.

[6] M. Johansson and A. Rantzer. Computation of piecewise quadratic Lyapunov functions for hybrid systems. IEEE Transactions on Automatic Control, 43:555-559, 1998.

[7] T. Hu and Z. Lin. Composite quadratic Lyapunov functions for constrained control systems. IEEE Transactions on Automatic Control, 48(3):440-450, 2003.

[8] J. C. Geromel and P. Colaneri. Robust stability of time varying polytopic systems. Systems and Control Letters, 55(1):81-85, 2006.

[9] R. K. Brayton and C. H. Tong. Stability of dynamical systems: a constructive approach. IEEE Transactions on Circuits and Systems, 26:224-234, 1979.

[10] F. Blanchini. Nonquadratic Lyapunov functions for robust control. Automatica, 31:451-461, 1995.

[11] F. Blanchini and S. Miani. A new class of universal Lyapunov functions for the control of uncertain linear systems. IEEE Transactions on Automatic Control, 44(3):641-647, 1999.

[12] A. L. Zelentsovsky. Nonquadratic Lyapunov functions for robust stability analysis of linear uncertain systems. IEEE Transactions on Automatic Control, 39(1):135-138, 1994.

[13] G. Chesi. LMI conditions for time-varying uncertain systems can be non-conservative. Automatica, 47(3):621-624, 2011. 
[14] G. Chesi, P. Colaneri, J. C. Geromel, R. H. Middleton, and R. Shorten. A nonconservative LMI condition for stability of switched systems with guaranteed dwell time. IEEE Transactions on Automatic Control, 57(5):1297-1302, 2012.

[15] G. Chesi. Sufficient and necessary LMI conditions for robust stability of rationally time-varying uncertain systems. IEEE Transactions on Automatic Control, 58(6):1546-1551, 2013.

[16] M. C. de Oliveira, J. Bernussou, and J. C. Geromel. A new discrete-time robust stability condition. Systems and Control Letters, 37:261-265, 1999 .

[17] V. J. S. Leite and P. L. D. Peres. An improved LMI condition for robust $D$-stability of uncertain polytopic systems. IEEE Transactions on Automatic Control, 48(3):500-504, 2003.

[18] E. N. Goncalves, R. M. Palhares, R. H. C. Takahashi, and R. C. Mesquita. New strategy for robust stability analysis of discrete-time uncertain systems. Systems and Control Letters, 56(7):516-524, 2007.

[19] M. E. Magana and S. H. Zak. Robust output feedback stabilization of discrete-time uncertain dynamical systems. IEEE Transactions on Automatic Control, 33:1082-1085, 1988.

[20] F. Blanchini. Ultimate boundeness control for uncertain discrete-time systems via set-induced Lyapunov functions. IEEE Transactions on Automatic Control, 39(2):428-433, 1994.

[21] J. Daafouz and J. Bernussou. Parameter dependent Lyapunov functions for discrete time systems with time varying parametric uncertainties. Systems and Control Letters, 43(5):355-359, 2001.

[22] G. Chesi. A Gram-SOS approach for robust stability analysis of discrete-time systems with time-varying uncertainty. In American Control Conference, pages 5883-5888, Washington, DC, 2013.

[23] F. Blanchini and S. Miani. Set-Theoretic Methods in Control. Birkhauser, 2008.

[24] G. Chesi, A. Tesi, A. Vicino, and R. Genesio. On convexification of some minimum distance problems. In European Control Conference, Karlsruhe, Germany, 1999. 
[25] P. A. Parrilo. Structured semidefinite programs and semialgebraic geometry methods in robustness and optimization. $\mathrm{PhD}$ thesis, California Institute of Technology, 2000.

[26] J.-B. Lasserre. Global optimization with polynomials and the problem of moments. SIAM Journal of Optimization, 11(3):796-817, 2001.

[27] M. Kojima. Sums of squares relaxations of polynomial semidefinite programs. Technical report, Tokyo Institute of Technology, 2003.

[28] A. Papachristodoulou and S. Prajna. A tutorial on sum of squares techniques for system analysis. In American Control Conference, pages 2686-2700, Portland, Oregon, 2005.

[29] G. Chesi. LMI techniques for optimization over polynomials in control: a survey. IEEE Transactions on Automatic Control, 55(11):2500-2510, 2010 .

[30] G. Stengle. A nullstellensatz and a positivstellensatz in semialgebraic geometry. Math. Ann., 207:87-97, 1974.

[31] M. Putinar. Positive polynomials on compact semi-algebraic sets. Ind. Univ. Math., 42:969-984, 1993.

[32] C. W. Scherer and C. W. J. Hol. Matrix sum-of-squares relaxations for robust semi-definite programs. Mathematical Programming Series B, 107(1-2):189-211, 2006.

[33] G. Hardy, J. E. Littlewood, and G. Pólya. Inequalities. Cambridge University Press, Cambridge, 1988.

[34] C. Scheiderer. Positivity and sums of squares: A guide to some recent results. In M. Putinar and S. Sullivant, editors, Emerging Applications of Algebraic Geometry, Vol. 149 of IMA Volumes in Mathematics and its Applications, pages 271-324. Springer, 2009.

[35] J. F. Sturm. Using SeDuMi 1.02, a MATLAB toolbox for optimization over symmetric cones. Optimization Methods and Software, 11-12:625653, 1999. 